\title{
Pre-operative predictors of knee range of motion during stair walking after total knee replacement
}

\author{
M. Van Der Linden1, P. Roche1, P. Rowe2, R. Nutton3. 1School \\ of Health Sciences, Queen Margaret University; 2 Health \\ $Q$ west, University of Strathclyde; 3Orthopaedic Department, \\ Royal Infirmary Edinburgh, UK
}

Summary: Hierarchical Multiple Regression was performed on the pre and postoperative measures of 56 patients with end-stage osteoarthritis. Post-operative range of knee motion during stair ascent and descent was predicted by pre-operative measures in different domain blocks.

Conclusions: Pre-operative variables predicting post-operative stair walking differ between stair ascent and descent. This has consequences for the treatment of patients waiting for a total knee replacement.

Introduction: In Scotland alone, over 3000 people receive a primary total knee replacement each year. This number is likely to rise over the next decade as a result of increasing life expectancy and increasing obesity. This study aimed to identify those preoperative factors predicting the range of motion used during stair ascent and descent one year after Total Knee Replacement surgery.

Patients/Materials and Methods: The pre-operative and 1 year post-operative results of fifty six patients ( 27 female, 29 male) with osteoarthritis were analysed. Average age was 69 years (range 38-87). Range of knee motion during stair ascent and descent was assessed using flexible electrogoniometry. Preoperative measures were grouped in three different domains.

Demographic domain age and Body Mass Index (BMI). Body Function domain: active range of knee motion in long sitting (ROMsit), Knee extensor moment (Mext), Pain on a Visual Analogue Scale and the stiffness component of the Western Ontario McMaster University Osteoarthritis Index (WOMAC).

Psychosocial Domain: The Tampa scale for 'fear of movement' (TSK) [1) and the sense of helplessness due to pain [2]. Hierarchical Multiple Regression was used to analyse the relative importance of measures grouped into the three domain blocks on range of motion of the operated knee during stair ascent (ROMup) and descent (ROMdown). Model 1 contained domain block 1, model 2 included domain blocks 1 and 2 and model 3 included domain blocks 1, 2 and 3. Statistical calculations were carried out using SPSS vs 15.

Results: Significant correlations $(\mathrm{p}<0.01)$ were found between ROMdown and ROMsit $(\mathrm{r}=0.31)$ and learned helplessness $(\mathrm{r}=-0.30)$ and between ROMup and age $(\mathrm{r}=-0.391)$, ROMsit $(\mathrm{r}=0.511)$ and Mext $(\mathrm{r}=0.348)$. Table 1 shows that in these models learned helplessness was a significant predicting variable for stair descent (beta $-0.538, p=0.025$ ) while for stair ascent, age (beta $0.375, p=0.005$ ) and ROMsit (beta $0.365, \mathrm{p}=0.021$ ) were significant predicting variables. Table 1 shows the coefficient of determination (R2), the significance of the change due to addition of the pre-operative measures in the next model and the significance of the model, for 
postoperative stair descent and ascent.

Discussion: These results show that postoperative stair ascent and descent are predicted by different pre-operative factors. For stair ascent the demographic factors age and function factor ROM are important, while for stair descent, only the addition of the psychosocial factors in model 3 resulted in a significant change. These results indicate that treatment of patients with end-stage osteoarthritis should not only be aimed at improving range of motion of the knee but should also take into account psychosocial variables such as a sense of helplessness due to pain.

Table 1

Stair descent

$\begin{array}{ccccccc}\text { model R2 } & \mathrm{p} \text { (change) } & \mathrm{p} \text { (model) } & \mathrm{R} 2 & \mathrm{p} \text { (change) } & \mathrm{p} \text { (model) } \\ 1 & 0.041 & 0.387 & 0.387 & 0.190 & 0.009 & 0.009 \\ 2 & 0.128 & 0.407 & 0.435 & 0.424 & 0.006 & 0.001 \\ 3 & 0.253 & 0.049 & 0.141 & 0.478 & 0.149 & 0.001\end{array}$

\section{References}

[1] Goubert L, et al. Clin J Pain 2004; 20: 103-10.

[2] DeVellis RF, Callahan LF. J Rheumatol 1993; 20: 866-9. 